\title{
A TWO-PARAMETER HOMOGENEOUS MEAN VALUE ${ }^{1,2}$
}

\section{D. TOBEY}

1. Introduction. The mean of order $t$ of the positive values $x=\left(x_{1}, x_{2}, \cdots, x_{n}\right)$ with positive weights $w=\left(w_{1}, w_{2}, \cdots, w_{n}\right)$, $\sum w_{i}=1$, is defined [3], [5] by

$$
\begin{aligned}
& M_{t}(x, w)=\left[\sum_{i=1}^{n} w_{i} x_{i}^{t}\right]^{1 / t}, \quad t \neq 0, \\
& M_{0}(x, w)=\prod_{i=1}^{n} x_{i}^{w_{i}}=\lim _{t \rightarrow 0} M_{t}(x, w) .
\end{aligned}
$$

Homogeneity in $x$ distinguishes $M_{t}$ from all other means of the form $\phi^{-1}\left[\sum_{i=1}^{n} w_{i} \phi\left(x_{i}\right)\right]$, where $\phi$ is any function with a unique inverse $\phi^{-1}[5$, Theorem 84].

Without losing homogeneity, $M_{t}$ has been generalized to the hypergeometric mean value $M(t, c ; x ; w)$ [4]. In the present paper we shall make a further generalization while maintaining homogeneity. We construct a two-parameter mean, $L(s, t ; x)$, by first forming the mean $M_{s}(x, u)$. Using an arbitrary weight function, $P(u)$, we then take an integral average over all possible choices of the weights $u$ satisfying $\sum_{i=1}^{n} u_{i}=1$. Because $u_{n}=1-u_{1}-\cdots-u_{n-1}$, the average requires an $(n-1)$-fold integration with respect to $u_{1}, u_{2}, \cdots, u_{n-1}$. If the variables $x$ are all positive, then for any real $s$ and $t$ we define

$$
\begin{aligned}
& L(s, t ; x)=\left[\int_{E} M_{s}^{t}(x, u) P(u) d u^{\prime}\right]^{1 / t}, \quad t \neq 0, \\
& L(s, 0 ; x)=\lim _{t \rightarrow 0} L(s, t ; x),
\end{aligned}
$$

where $u^{\prime}=\left(u_{1}, \cdots, u_{n-1}\right), d u^{\prime}=d u_{1} d u_{2} \cdots d u_{n-1}, P(u) \geqq 0, \int_{E} P(u) d u^{\prime}$ $=1$ and $E=\left\{u^{\prime} \mid u_{i}>0,1 \leqq i \leqq n-1\right.$ and $\left.u_{n}=1-u_{1}-\cdots-u_{n-1}>0\right\}$.

The $L(s, t ; x)$ mean is homogeneous in $x$. It can be regarded as a special case of the integral mean [5, Chapter 3]

Received by the editors May 31, 1966

1 Work was performed in the Ames Laboratory of the USAEC and supported in part by the National Aeronautics and Space Administration under Grant NsG-293 to Iowa State University.

2 This report is based on a Ph.D. thesis submitted May 1966 to Iowa State University. The author wishes to thank Professor B. C. Carlson who not only directed the thesis research but also has aided in preparing this paper for publication. 


$$
M_{t}(f, P)=\left[\int f^{t}(u) P(u) d u\right]^{1 / t},
$$

but it is a generalization of previously known ways of constructing a homogeneous mean of the discrete variables $x_{1}, x_{2}, \cdots, x_{n}$. After defining a set of natural weights $w$ associated with the function $P(u)$, we shall show (Theorem 3 ) that $L(s, t ; x)$ contains $M_{t}(x, w)$ in the special case $s=t$. It contains also the hypergeometric mean, $M(t, c ; x ; w)$, in the special case $s=1$ and $P(u)=P(c w ; u)$, a particular weight function depending on the parameters $c w=\left(c w_{1}, c w_{2}, \cdots, c w_{n}\right)$ [4, Equation (2.2)]. All properties of $M(t, c ; x ; w)$ which do not depend explicitly on $c$ can be generalized to properties of $L(s, t ; x)$. In this generalization, $s$ and $t$ each play roles analogous to that of $t$ in $M(t, c ; x ; w)$.

2. Elementary properties of $L(s, t ; x)$. If $P(u)$ is such that $L^{t}(s, t ; x)$ is an improper integral, it is easily shown to converge uniformly in $s, t$, and $x$ for $0<m \leqq x_{i} \leqq M, 1 \leqq i \leqq n$, all real $s$, and $-T \leqq t \leqq T$. As a result of this uniform convergence and the continuity of $M_{s}^{t}(x, u)$ in $s, t, x$, and $u, L(s, t ; x)$ is continuous in $s, t$, and $x$.

In considering some limiting values of $L(s, t ; x)$, we use the notation $x_{\max }=\max \left\{x_{1}, x_{2}, \cdots, x_{n}\right\}$ and $x_{\min }=\min \left\{x_{1}, x_{2}, \cdots, x_{n}\right\}$.

THEOREM 1.
(a) $L(s, 0$;
$x)=\lim _{t \rightarrow 0}$
$t ; x)=x_{\operatorname{rax}}$
$L(s, t ; x)=\exp \left[\int_{E} \ln M_{s}(x, u) P(u) d u^{\prime}\right]$
(b) $\lim _{t \rightarrow \infty} L(s, t ; x)=x_{\text {raxax }}$,
(c) $\lim _{t \rightarrow-\infty} L(s, t ; x)=x_{\min }$,
(d) $\lim _{s \rightarrow \infty} L(s, t ; x)=x_{\max }$,
(e) $\lim _{s \rightarrow-\infty} L(s, t ; x)=x_{\min }$.

Proof. Part (a) is an application of L'Hôpital's rule, differentiation with respect to $t$ under the integral sign being permissible because the integral of the derivative converges uniformly for $-T \leqq t$ $\leqq T$ and $0<m \leqq x_{i} \leqq M, 1 \leqq i \leqq n$. Parts (b) and (c) follow from properties of the integral mean $M_{t}(f, p)\left[5\right.$, p. 143] with $f(u)=M_{s}(x, u)$. Parts (d) and (e) follow from properties of $M_{s}(x, u)$ [5, Theorem 4].

TheOREM 2. (a) $L(s, t ; x)$ is a strictly increasing function of $x$; i.e. if $x_{i} \leqq y_{i}$ for all $i$ and $x_{j}<y_{j}$ for some $j$, then $L(s, t ; x)<L(s, t ; y)$.

(b) If $x_{\max }>x_{\min }$, then $L(s, t ; x)$ is a strictly increasing function of $t$.

(c) If $x_{\max }>x_{\min }$, then $L(s, t ; x)$ is a strictly increasing function of $s$.

Proof. (a) From the definition of $M_{s}(x, u)$ we see that $M_{s}(x, u)$ 
$<M_{s}(y, u)$. The result is evident by inspection of (1.2) and Theorem 1(a). Part (b) is a property of the integral mean $M_{t}(f, P)[5$, p. 144] with $f(u)=M_{s}(x, u)$. For (c) it suffices to observe that $f=M_{s}(x, u)$ is a strictly increasing function of $s[5$, Theorem 16] and that $M_{t}(g, P)>M_{t}(f, P)$ if $g(u)>f(u)$ for all $u$.

The following theorem shows that the elementary mean $M_{s}(x, w)$ is a special case of the $L(s, t ; x)$ mean; the weights $w=\left(w_{1}, w_{2}, \cdots, w_{n}\right)$ are the "natural weights" associated with the weight function $P(u)$. We define the natural weights by

$$
w_{i}=\int_{E} u_{i} P(u) d u^{\prime}, \quad 1 \leqq i \leqq n .
$$

Theorem 3. If $w$ denotes the natural weights, then $L(s, s ; x)$ $=M_{s}(x, w)$.

Proof. If $s \neq 0$,

$$
\begin{aligned}
L(s, s ; " x)] & =\left[\int_{E} M_{s}^{s}(x, u) P(u) d u^{\prime}\right]^{1 / s}=\left[\int_{E} \sum_{i=1}^{n} u_{i} x_{i}^{8} P(u) d u^{\prime}\right]^{1 / s} \\
& =\left[\sum_{i=1}^{n} x_{i}^{s} \int_{E} u_{i} P(u) d u^{\prime}\right]^{1 / s}=M_{s}(x, w) .
\end{aligned}
$$

If $s=0$,

$$
\begin{aligned}
L(0,0 ; x) & =\exp \left[\int_{E} \ln \prod_{i=1}^{n} x_{i}^{u_{i}} P(u) d u^{\prime}\right] \\
& =\exp \left[\sum_{i=1}^{n} \ln x_{i} \int_{E} u_{i} P(u) d u^{\prime}\right] \\
& =\exp \left[\ln \prod_{i=1}^{n} x_{i}^{w_{i}}\right]=M_{0}(x, w) .
\end{aligned}
$$

A given set of weights $w$ occurs as the natural weights associated with a large class of functions $P$. This class contains the family $P(c w ; u)[4$, Equation $(2.2)]$ where the natural weights are just the parameters $w$. If $P(u)=P(c w ; u), L(s, t ; x)$ becomes the "generalized hypergeometric mean" $L(s, t, c ; x ; w)$. In particular, $L(1, t, c ; x ; w)$ $=M(t, c ; x ; w)$. For $s \neq 0, L(s, t, c ; x ; w)$ can be expressed in terms of $M(t, c ; x ; w)$ by using the identity

$$
\begin{aligned}
L^{r}(s, t ; x) & =\left[\int_{E}\left[\sum_{i=1}^{n} u_{i}\left(x_{i}^{r}\right)^{s / r}\right]^{(t / r) /(s / r)} P(u) d u^{\prime}\right]^{r / t} \\
& =L\left(s / r, t / r ; x^{r}\right), \quad r, s, t \neq 0 .
\end{aligned}
$$


A similar argument gives the same identity if $s$ or $t$ is zero. Putting $r=s$ and $P(u)=P\left(c w ; u^{\prime}\right)$, we have

$$
L(s, t, c ; x ; w)=\left[M\left(t / s, c ; x^{s} ; w\right)\right]^{1 / s} .
$$

Although Theorems 1 and 2 show at once that $x_{\min }<L(s, t ; x)$ $<x_{\max }$, the introduction of natural weights allows us to give sharper inequalities:

Corollary 1. Let $w$ denote the natural weights. If $x_{\max }>x_{\min }$ and $s<t$, then $M_{s}(x, w)<L(s, t ; x)<M_{t}(x, w)$. The inequalities are reversed if $s>t$.

Proof. By Theorem $2, L(s, t ; x)$ is an increasing function of each of the parameters $s$ and $t$. Hence, applying Theorem 3 , if $s<t$,

$$
M_{s}(x, w)=L(s, s ; x)<L(s, t ; x)<L(t, t ; x)=M_{t}(x, w),
$$

with reversed inequalities if $s>t$.

It is well known [1, p. 9] that if $\log f(r, u)$ is convex in $r$, then $\log \int f(r, u) d u$ is convex in $r$. To study the convexity of $L^{s}(s, t ; x)$ in $s$, we need the analogous theorem that if $r \log f(r, u)$ is convex in $r$, then $r \log \int f(r, u) d u$ is convex in $r$ for $r>0$.

LEMMA 1. Let $f^{r}(r, u)$ be continuous in $r$ and $u$ and log convex in $r$. Then, if $\left[\int_{E} f(r, u) P(u) d u^{\prime}\right] r$ is continuous, it is log convex in $r$ for $r>0$.

Proof. If $f^{r}(r, u)$ is continuous and log convex in $r$, then $\left[f^{r}(r, u)\right]^{1 / r}$ $=f(r, u)$ is continuous and log convex in the variable $1 / r$ for $r>0$ [5, Theorem 119]. Since $f(r, u)$ is continuous in $u$ and $\log$ convex in $1 / r, \int_{E} f(r, u) P(u) d u^{\prime}$ is log convex in $1 / r[1, p .9]$. Hence $\left[\int_{E} f(r, u) P(u) d u^{\prime}\right] r$ is log convex in $r$ for $r>0$ [5, Theorem 119].

ThEOREM 4. (a) $L^{s}(s, t ; x)$ is log convex in $s$ for $t / s \geqq 0$. (b) $L^{t}(s, t \cdot x)$ is log convex in $t$.

Proof. (a) For any fixed $t \neq 0, L^{s}(s, t ; x)=\left[\int_{E} M_{s}^{t}(x, u) P(u) d u^{\prime}\right]^{s / t}$ $=\left[\int_{E} M_{r}(y, u) P(u) d u^{\prime}\right] r$, where $y_{i}=x_{i}^{t}, 1 \leqq i \leqq n$, and $r=s / t$. Since $M_{r}^{r}(y, u)$ is log convex in $r$ [5, Theorem 87], Lemma 1 implies $\left[\int_{E} M_{r}(y, u) P(u) d u^{\prime}\right] r$ is $\log$ convex in $r$ for $r=s / t>0$. But if a function is $\log$ convex in $r$, it is log convex in $t r=s$ for any fixed $t \neq 0,[1$, Theorem 1.10].

For $t=0, \ln L^{s}(s, 0 ; x)=\int_{E} \ln M_{s}^{s}(x, u) P(u) d u^{\prime}$ is convex in since $M_{s}^{s}(x, u)$ is log convex in $s$.

(b) $L^{t}(s, t ; x)$ is log convex in $t$ since $M_{t}^{t}(f, P)$ is log convex in $t$ [5, Theorem 197], and $L^{t}(s, t ; x)$ has this form with $f(u)=M_{s}(x, u)$.

3. Inequalities for $L(s, t ; x)$. The next two theorems are results of 
properties of the mean $M_{s}(x, u)$ and the integral mean $M_{t}(f, P)$. The first comes from Theorems 24, 186 and 198 of [5]. The second comes from Theorems 12 and 188 of [5], with the special case $t=0$ as an elementary result of properties of the logarithm. We use the notation $x+y=\left(x_{1}+y_{1}, x_{2}+y_{2}, \cdots, x_{n}+y_{n}\right)$ and $x y=\left(x_{1} y_{1}, x_{2} y_{2}, \cdots, x_{n} y_{n}\right)$.

ThEOREM 5 (MINKowsKI). Let $x$ and $y$ be vectors with $x_{i}>0$ and $y_{i}>0,1 \leqq i \leqq n$. Then, unless $s=t=1$ or $x_{i}=k y_{i}, 1 \leqq i \leqq n$,

$$
L(s, t ; x+y)<L(s, t ; x)+L(s, t ; y), \quad(s, t \geqq 1),
$$

with reversed inequality if $s, t \leqq 1$. Equality holds in the exceptional cases.

Theorem 6 (HöLDER). Let $x$ and $y$ be vectors with $x_{i}>0$ and $y_{i}>0$, $1 \leqq i \leqq n$, and let $p$ and $q$ be real numbers greater than unity such that $1 / p+1 / q=1$. Then, unless $s=t=0$ or $x_{i}^{p}=k y_{i}^{q}, 1 \leqq i \leqq n$,

$$
L(s, t ; x y)<L^{1 / p}\left(s, t ; x^{p}\right) L^{1 / q}\left(s, t ; y^{q}\right), \quad(s, t \geqq 0),
$$

with reversed inequality if $s, t \leqq 0$. Equality holds in the exceptional cases.

By defining the mean of a matrix of values $x_{i j}$, both of the preceding theorems can be included in an analogue of the Jessen-Ingham inequality [5, Theorems 26 and 203]. The proof $[7$, p. 20] relies primarily on the Minkowski inequality and uses Hölder's inequality for a special case.

Finally, we shall show that $L(s, t ; x)$ satisfies a Kantorovich inequality [2, p. 208]. The proof proceeds by adapting a method due to Rennie [6, p. 982].

Theorem 7 (Rennie). Let $0<A \leqq x_{i} \leqq B, 1 \leqq i \leqq n$. Then if $t \neq 0$,

$$
\begin{aligned}
L^{t}(s, t ; x)+A^{t} B^{t} L^{-t}(s,-t ; x)= & L^{t}(s, t ; x) \\
& +A^{t} B^{t} L^{t}(-s, t ; 1 / x) \leqq A^{t}+B^{t},
\end{aligned}
$$

with equality if and only if $x_{i}=A$ or $x_{i}=B, 1 \leqq i \leqq n$.

Proof. The equality between the first and second members is seen by (2.2) with $r=-1$. To obtain the inequality we notice that $M_{s}^{t}(x, u)$ is bounded between $A^{t}$ and $B^{t}$ and, following Rennie, we consider

$$
\left[M_{s}^{t}(x, u)-A^{t}\right]\left[1-B^{t} M_{s}^{-t}(x, u)\right] P(u) \leqq 0,(t \neq 0) .
$$

Integrating and rearranging, we have

$$
\int_{B} M_{*}^{t}(x, u) P(u) d u^{\prime}+A^{t} B^{t} \int M_{*}^{-t}(x, u) P(u) d u^{\prime} \leqq A^{t}+B^{t} .
$$


Equality holds if and only if $M_{s}^{t}(x, u)=A^{t}$ or $M_{s}^{t}(x, u)=B^{t}$ for all $u \in E$.

Theorem 8 (Kantorovich). If $t>0$ and $0<A \leqq x_{i} \leqq B, 1 \leqq i \leqq n$, then

$$
\begin{aligned}
1 & \leqq L(s, t ; x) / L(s,-t ; x)=L(-s, t ; x) L(s, t ; 1 / x) \\
& \leqq\left[\left(A^{t}+B^{t}\right) / 2\right]^{1 / t}\left[\left(A^{-t}+B^{-t}\right) / 2\right]^{1 / t},
\end{aligned}
$$

with equality on the left if and only if $x_{\max }=x_{\min }$, and equality on the right if and only if $A=B$.

Proof. The left inequality holds because $L(s, t ; x)$ is a strictly increasing function of $t$ by Theorem $2(\mathrm{~b})$, unless $x_{\max }=x_{\min }$. The equality between the second and third members is due to (2.2) with $r=-1$. To obtain the right-hand inequality we start with Rennie's inequality (Theorem 7),

$$
L^{t}(s, t ; x)+A^{t} B^{t} L^{-t}(s,-t ; x) \leqq A^{t}+B^{t} .
$$

Dividing by 2, applying the inequality of the arithmetic and geometric means to the left side, and squaring, we find

$$
\begin{aligned}
{[L(s, t ; x) / L(s,-t ; x)]^{t} } & \leqq\left(A^{t}+B^{t}\right)^{2} / 4 A^{t} B^{t} \\
& =\left[\left(A^{t}+B^{t}\right) / 2\right]\left[\left(A^{-t}+B^{-t}\right) / 2\right] .
\end{aligned}
$$

Taking the $t$ th root gives the desired inequality. If $A=B$, we have equality at each step of the proof; if $A \neq B$, then the conditions for equality in Theorem 7 imply strict inequality of the arithmetic and geometric means.

\section{REFERENCES}

1. E. Artin, The gamma function, Holt, Rinehart and Winston, New York, 1964.

2. E. F. Beckenbach, On the inequality of Kantorovich, Amer. Math. Monthly 71 (1964), 606-619.

3. E. F. Beckenbach and R. Bellman, Inequalities, Ergebnisse der Mathematik, Springer, Berlin, 1961.

4. B. C. Carlson, A hypergeometric mean value, Proc. Amer. Math. Soc. 16 (1965), 759-766.

5. G. H. Hardy, J. E. Littlewood and G. Pólya, Inequalities, 2nd ed., Cambridge Univ. Press, Cambridge, 1959.

6. B. C. Rennie, An inequality which includes that of Kantorovich, Amer. Math Monthly 70 (1963), 982.

7. M. D. Tobey, Ph.D. thesis, Iowa State University, Ames, 1966 (unpublished).

\section{Iowa State University}

